\title{
III. On torsional oscillations of wires
}

\section{Dr. W. Peddie}

To cite this article: Dr. W. Peddie (1894) III. On torsional oscillations of wires, Philosophical Magazine Series 5, 38:230, 36-55, DOI: 10.1080/14786449408620600

To link to this article: http://dx.doi.org/10.1080/14786449408620600

$$
\text { 曲 Published online: } 08 \text { May } 2009 .
$$

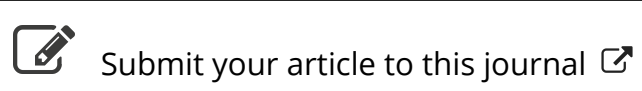

\footnotetext{
Џll Article views: 3
}

Q View related articles $\sqsubset$ 


\section{[ 36 ]}

III. On Torsional Oscillations of Wires. By Dr. W. Pedde, Physical Laboratory, Edinlurgh University*.

\section{Sketch of Previous Work $\uparrow$.}

TTHE present subject is part of the more general one-the deformations of a non-rigid solid. That strain, or part of a strain, which disappears wholly on the removal of the distorting stress, is called temporary strain: that which is observed after the complete removal of the distorting stress, is called permanent strain or set, though it may, and usually does, diminish as the time which has elapsed since the complete removal of distorting stress increases. The latter effect, together with the converse effect of the gradual increase of set under continued constant stress, is called after-action by the Germans.

In 1835 (Pogg. Ann.) Weber investigated the laws of permanent set of a stretched fibre. In 1837 (B. A. Report; see also B. A. Reports, 1843, 1844) Hodgkinson, as the result of experiments on cast iron, came to the conclusion that "the maxim of loading bodies within the elastic limit has no foundation in nature" ; that is, permanent set is produced by any stress however small. In 1842 (Ann. de Chim. et Phys.) and 1848 (Pogg. Ann. Ergbd. ii.) Wertheim observed that permanent set occurred in a previously undistorted body as the result of any stress however small ; and in 1848 (Camb. \& Dubl. Math. Journ.) this limitation to Hodgkinson's statement was independently pointed out, as a deduction from theory, by Prof. James Thomson. On the other hand, a body previously distorted in a given sense may be again distorted to a smaller, or the same, extent in the same sense without the production of new permanent set.

Between 1858 and 1862 G. Wiedemann made statical experiments on the torsion of rods, in the course of which he verified Wertheim's observation; and in 1880 (Phil. Mag. vol.ix.) he published the results of more extended experiments of the same kind. One of these results is that, after repeated twistings, alternately in opposite directions, by a given couple, the set of a rod becomes constant; and, if the rod be again twisted, by increasing couples, in the direction of the last twist, the strain (measured from the position of set) is

* Communicated by the Author, having been read before the Royal Society of Ediaburgh, December 18, 1893.

$\dagger$ This sketch is a mere outline. Fuller references will be found in the papers quoted. 
practically proportional to the stress so long as the original value of the conple is not exceeded. Another is that the reversed couple produces a greater strain, measured from the last set, than does the equal direct couple; the difference in this case corresponds to the change of zero produced by the reversal of the couple- that is, to the set. Again, by repeated reversals of twist under a given couple, the total torsion and the set diminish to fixed minimum values. Also, in the case of torsion in one direction, the values of the total torsion and of the set increase at increasing rates as the couple increases, and the latter relatively at a greater rate than the former: these values for a given couple increase to maxima by repeated applications of the couple, and this increase is also relatively greater in the set than in the total torsion. Wiedemann remarks that the approach of the position of final set to that of final total torsion in this case is a phenomenon of the same kind as the narrowing of the limits of total torsion and of set by repeated reversals of a given couple; the only difference is that the negative couple is zero. He calls the process by which the wire is brought into the steady state as regards total torsion and set the process of accommodation.

In 1865 (Proc. Roy. Soe. Lond.) Lord Kelvin deseribed results obtained from the observation of torsional oscillations of wires. He discovered the phenomenon of "elastic fatigue," and found that the diminution of the range of oscillation, per equal number of oscillations, followed the law of compound interest when the range was very much smaller than the palpable limits of elasticity. Tomlinson's observations (Phil. Trans. 1886) support this conclusion.

\section{Present Observations and Results.}

So far as 1 am aware, no attempt has been made to find the law of decrease of the range of oscillation when it is so large that it is accompanied by marked set-set which may amount to a large fraction of the total range. Lord Kelvin's observations were purposely made upon small oscillations in order to avoid the disturbances which are introdnced when the oscillations are large. Because of the known intimate dependence of the instantaneons state of strain of a body under given stresses upon all the previons strains to which it has been subjected, it might be supposed that it would be absolutely impossible to deduce with certainty any general law of decay of large oscillations. In other words, any systematic arrangement of conditions might seem to be unattainable because of the possible intrusion of arbitrary and uncontrollable, perhaps even untraceable, conditions. As a matter of fact, I have 
found that, in all my observations hitherto made, such arbitrariness is notably absent; and I have been able to obtain an extremely accurate empirical formula for the representation of the results.

The results here given refer only to a single iron wire whose extremities were soldered into holes drilled axially in stout brass rods. The length of the wire was $89 \cdot 1$ centim., and its diameter was $0 \cdot 1011$ centim. The one rod was firmly clamped in a vertical position, with the wire suspended from it ; and to the other rod was attached, symmetrically and horizontally, a heavy lead ring of considerable moment of inertia. In performing the observations, one experimenter increased the torsional oscillations of the system up to a predetermined maximum, taking care to avoid as far as possible any swing of the system like that of an ordinary pendulum. Whenever the required maximum oscillation was attained, the system was left to itself, except in so far as any marked swing of the latter kind was damped out in such a way as not to interfere, by friction or otherwise, with the torsional oscillations. Another observer commenced at once to take readings of the maximum elongation by means of a telescope placed a few yards off. The scale was fastened round the outer circumference of the lead ring, and a fixed pointer was placed close in front of it. At first readings were taken at the end of each complete oscillation; subsequently, as the time-rate of decay of the oscillations became less, readings were taken at the end of two, three, five, or more, complete oscillations. A curve was then plotted with the scale-readings as ordinates and the number of swings as abscissæ. The oscillations were found to be almost isochronous, so that the axis of abscissæ was practically a time-axis. In almost all cases the curve showed traces of ordinary pendulum oscillations, but a smooth curve could easily be drawn on the average through the observed points so as to avoid all such irregularities. It would serve no useful purpose to give here the full details of each experiment. Their general nature will be seen from the curves shown in fig. 1, and the special data given in Table II. will be found sufficient for each. In fig. 1 the curves give the data obtained from observation, and the points show positions calculated from the respective equations in Table I.

It was found that equations of the form

$$
y^{n}(x+a)=b, \quad \text {. . . . . }
$$

where $a, b$, and $n$ are constants, applied with great accuracy in each case Table I. gives details on this point. 
The curves C, G, H, I, J, K are omitted as they were made with an entirely different object and the readings did not cover the same range.

The actual value of the constant added to $x$ depends upon the interval which elapses after starting the experiment until the first reading is taken. Thus the fact that in B this constant is greater by about unity than the similar constants in D, E, F, while the first reading in $\mathrm{B}$ is much less than the first readings in $\mathrm{D}, \mathrm{E}, \mathrm{F}$, points to the conclusion that the first reading in that curve was taken one oscillation later than the first readings in the latter. We should therefore expect that the value will be small when the initial range is large, as in M. The values of the other two constants in the equation for $M$ are much increased relatively to their values in the preceding equationsa fact which illastrates the dependence of the action at any stage upon the previous treatment of the wire. The decay of the oscillations is at first more rapid, afterwards more slow, than in the preceding experiments.

In $\mathrm{P}$ the phenomenon of elastic fatigue is very apparent. The conditions were practically the same in this experiment as in, for example, $\mathbf{E}, \mathbf{F}$, and $\mathrm{O}$, with the exception that in $\mathrm{P}$ the wire was kept oscillating to the maximum extent for about half an hour before the observations began. The rate of decay of the oscillations is immensely increased at all the observed values of the range.

Putting aside the special experiments $\mathrm{M}$ and $\mathrm{P}$, we find that, after the wire had once reached a steady state (in B), the steady state was maintained day after day; so that it was easy to repeat an experiment under practically the same conditions. Even the exceptional treatment in MI did not prevent the return of the wire to its old condition before the experiment on the following day was performed. In only one case, $L_{1}$, is there any exception, and this may have been due to a difference of temperature.

In the earlier experiments the initial range is said to be over 100. No exact record was kept, but the excess was considerable; the actual angle was probably about 125 . In the experiment $O$ the angle was maintained steadily at 125 for som $\theta$ time before the wire was let go and the observations were begun. The constants were not altered by this treatment; and this seems to indicate that the "after-action" under steady stress has little or no effect in these experiments, which are made under otherwise similar conditions.

The experiment $\mathrm{C}$ was performed on the same date as $\mathrm{B}$; so that $D$ was performed after the wire had been at rest for 


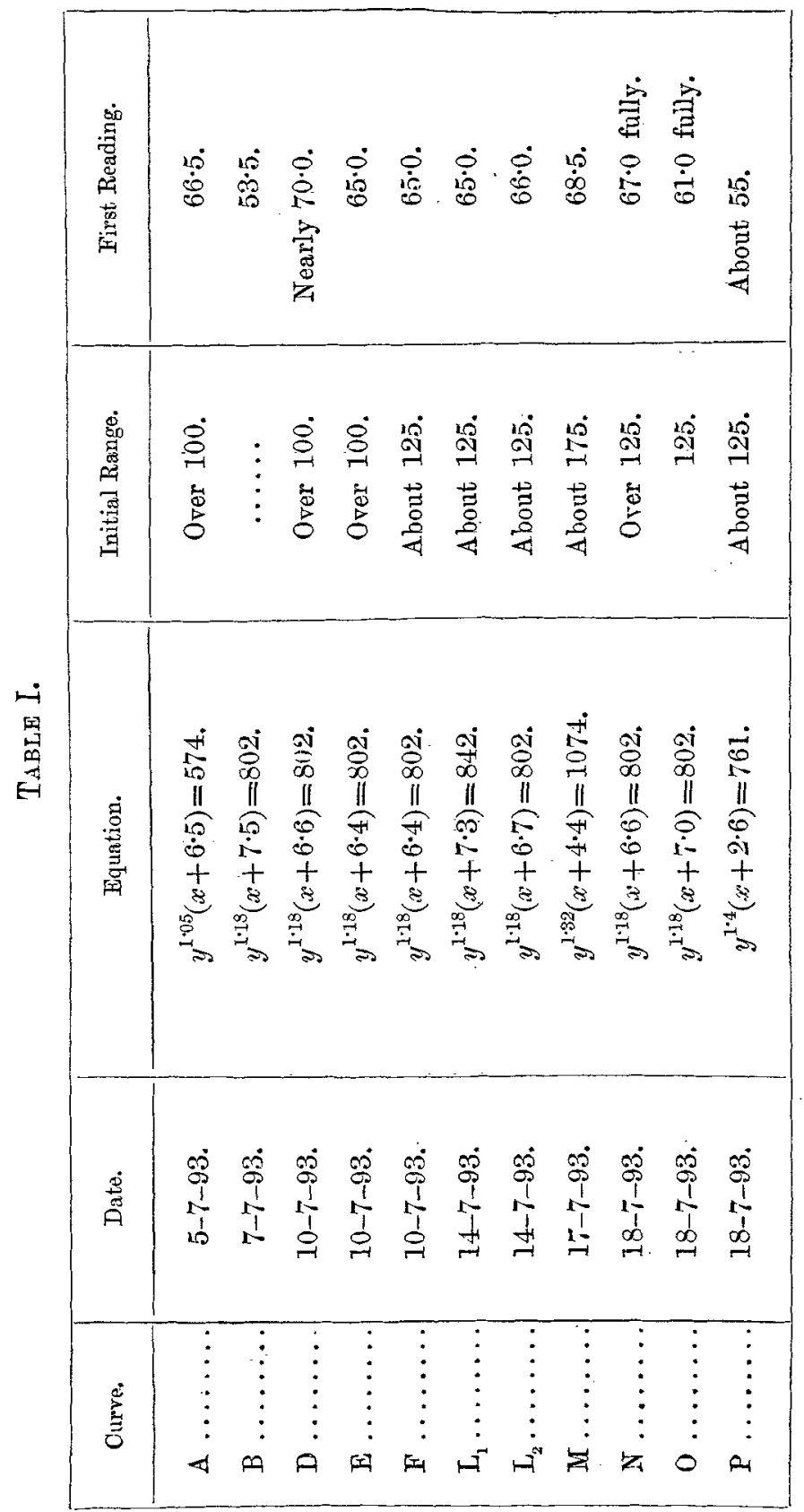




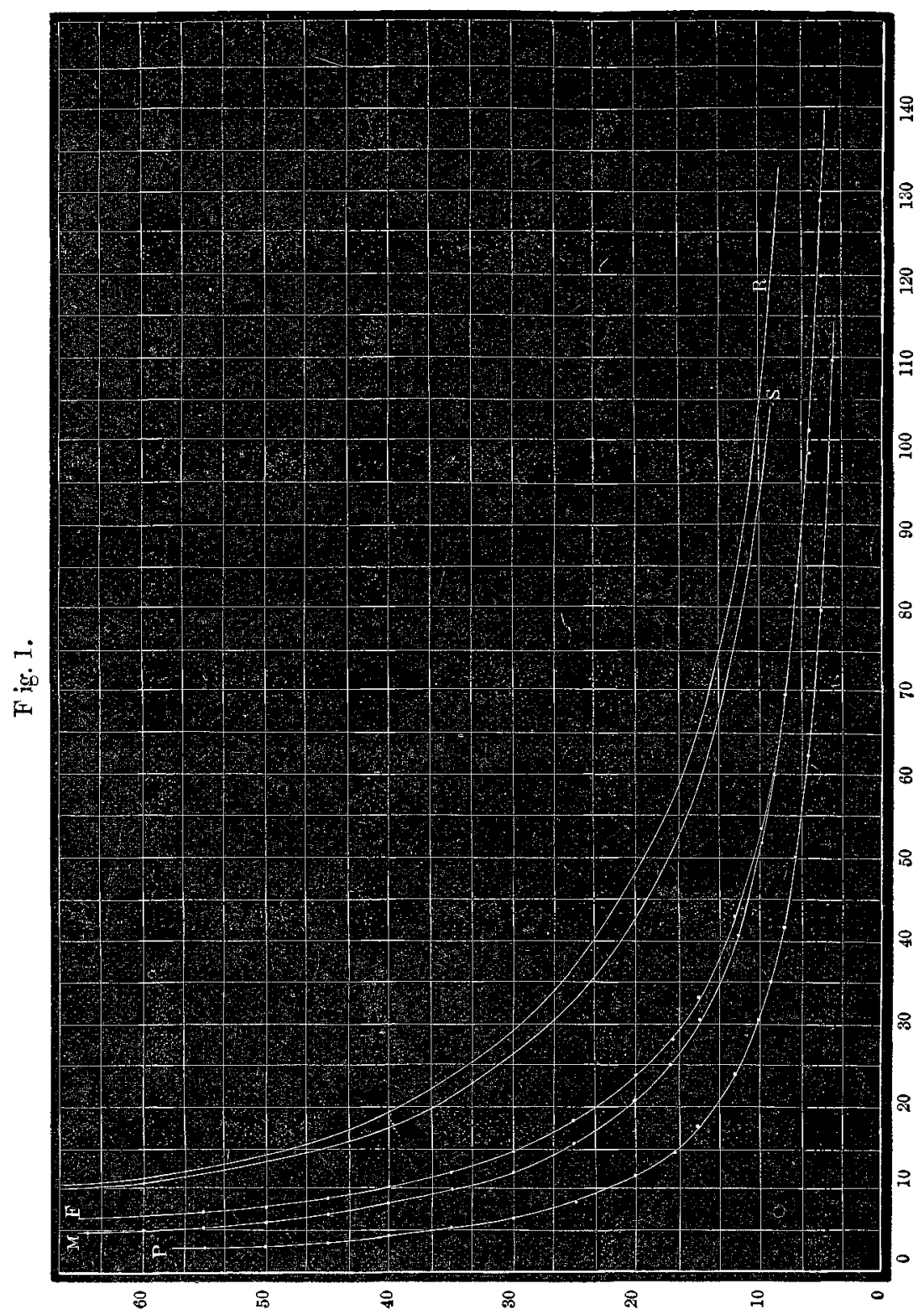


three days. Between the experiments $F$ and $L_{1}$ experiments were performed every day - twelve experiments in all, some with large, some with small, oscillations.

\section{Test of the Empirical Formulce.}

In the following table a comparison is made of observed results in each experiment with the results calculated from the formulæ. In the upper row are given values of $y$ common to all the experiments.

These figures show that the correspondence between the observed results and the results derived from the empirical formulæ is very close, and that it holds throughout a large range of values of $x$. The curves in fig. 1 show this even more clearly.

All the results given in Table II. for each experiment with the exception of $\mathrm{P}$ were observed within ten minutes after starting the oscillations. All the results given for $\mathrm{P}$ were observed within thirteen minutes. Within these time-limits, therefore, the quantity $n$ in the equation is practically constant. Yet, since from that table we see that the value of $n$ may diminish from 1.32 to 1.18 in the course of twenty-four hours, we cannot assume that $n$ will not vary in amount in any one experiment until the vibrations cease to be observable. As a matter of fact, it does so vary,

\section{Variations in the Value of $\mathrm{n}$.}

From Table I. it appears that when a fresh wire is oscillated with a given initial maximum range on different occasions, the quantity $n$ at first increases in magnitude and ultimately reaches a value which remains constant so long as the treatment of the wire from day to day is fairly uniform. This value is exceeded if the given initial range be exceeded, or if the wire be "fatigued" by long-continued oscillation; but the previous steady value of $n$ is soon reacquired.

If the empirical formula above used be correct, any two values of $y$ corresponding to a constant difference in $x$ must satisfy the condition

$$
\frac{y_{1}^{n}-y_{2}^{n}}{y_{1}^{n} y_{2}^{n}}=\text { constant. . . . . . }
$$

If too large a value of $n$ be used in this equation, the value of the quantity on the left-hand side will increase when the values of $y_{1}$ and $y_{2}$ decrease. If too small a value be used, the quantity will decrease when $y_{1}$ and $y_{2}$ decrease. 
Torsional Oscillations of Wires.

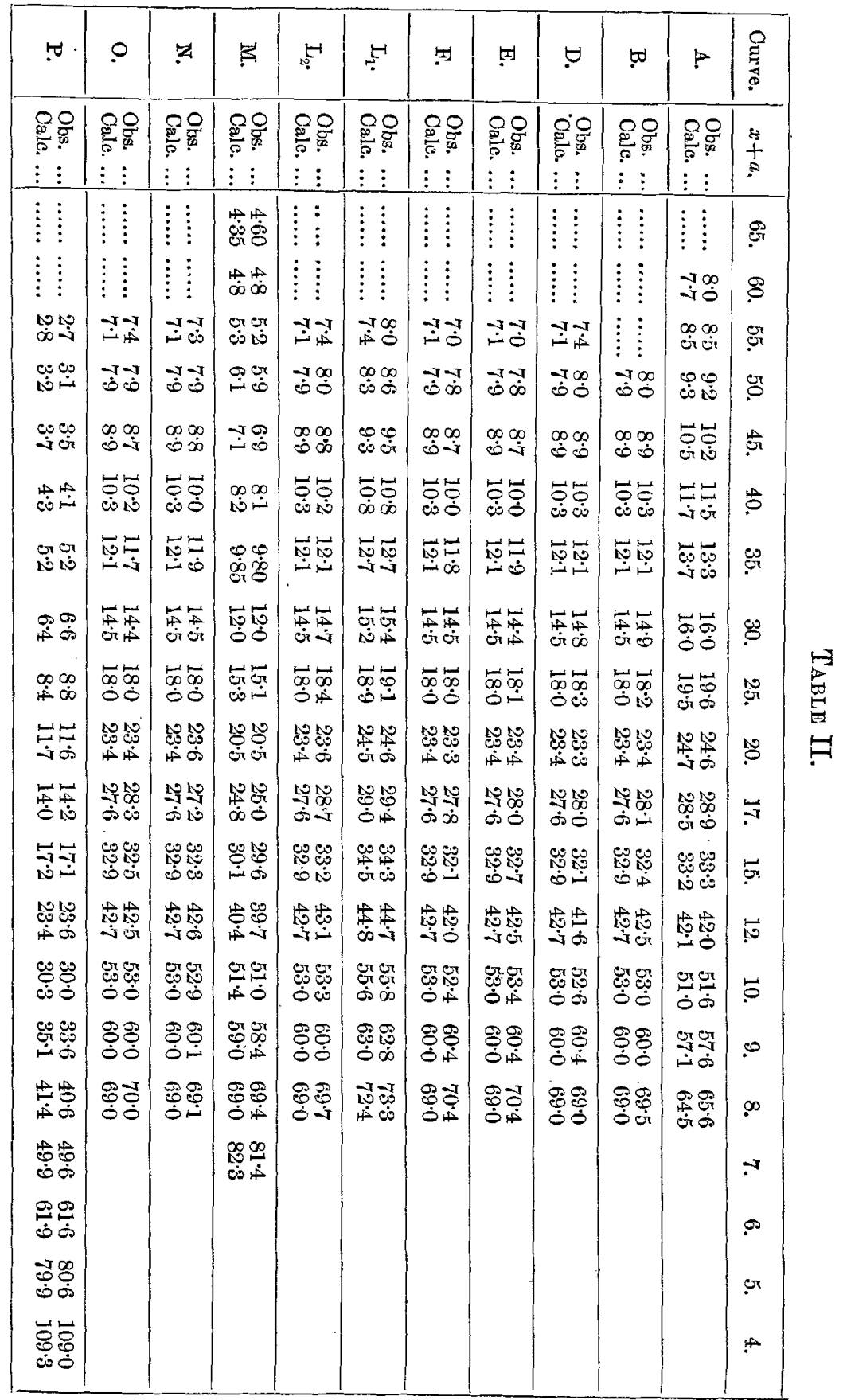


We shall apply this test to the results of experiment $\mathrm{C}$ (not given in Table I.), in which the initial range was only about 80 , and 300 oscillations were observed. The values of $y$ for which $x$ has the ralues $10,20,30,40$ are respectively $44 \cdot 9$, $31 \cdot 9,25 \cdot 3$, and $21 \cdot 1$. The values of the left-hand side of (2), with $n=1$, for the stretches of $x \cdot 10-20,20-30,30-40$, are respectively $0.0091,0.0082,0.0078$. Hence the true value of $n$ for this part of the curve is greater than unity. The values of $y$ for which $x$ has the values $80,160,220,300$ are respectively $13.5,7 \cdot 9,6,4 \cdot 4$. The values of the left-hand side of (2), with $n=1$, for the stretches of $x 80-160,220-300$, are respectively 0.052 and 0.061 . Hence, in this part of the curve, where the oscillations are small relatively to the initial range, the true value of $n$ is less than unity. If in this part of the curve we take $n=\cdot 05$, we get, instead of 0.052 and 0.061 , the quantities 0.083 and 0.068 respectively; so that $n$ exceeds 0.5 .

We shall next apply equation (2) to the results of experiments $R$ and $S$, performed at the dates 15-11-93 and 17-11-93 respectively. As in the experiments given in Table I., the initial range was about 125 . Previous to the former date the wire had been left at rest with the lead ring appended since the date 19-7-93, i.e. during a period of nearly four months.

In the curve $\mathbf{R}$ the values of $y$ for which $x$ has the values $10,20,30,40$ are respectively $38 \cdot 8,29,22 \cdot 9,18 \cdot 8$. The values of the left-band side of (2), for $n=1$, over the three stretches of $a 10-20,20-30,30-40$, are respectively 0.0087 , $0 \cdot 0092,0 \cdot 0095$. For $n=0.5$ the numbers are $0.0025,0.0024$, 0.0021 . Hence $n$ is greater than 0.5 and less than unity.

In the curve $\mathrm{S}$, at the same values of $x$, the values of $y$ are $36 \cdot 6,26 \cdot 9,21 \cdot 1,17 \cdot 3$; and the values of the left-hand side of (2) are $0.0098,0.0102,0.0104$. Hence $n$ is less than unity. The differences of these numbers are 0.00022 and 0.0002 . The corresponding differences in $\mathrm{R}$ are 0.0005 and 0.0003 . Hence $n$ is larger in $\mathrm{S}$ than in $\mathrm{R}$.

$$
\text { The "Compound Interest" Law. }
$$

There can be no doubt that, when the range of oscillation is " much smaller than the palpable limits of elasticity," the decrease of range per oscillation bears a constant ratio to the range itself. This was first shown by Lord Kelvin and, more recently, was confirmed by Tomlinson. When the range is increased this law no longer holds, the logarithmic decrement becoming larger.

In experiment $\mathrm{C}$ the observations were continued for a much longer time than in any other experiment, yet the stage 
at which the logarithmic decrement becomes constant was not reached. The values of $y$ for which $x$ had the values 80,120 , $160,180,200,220,260$, 300, were respectively $13 \cdot 5,10,7 \cdot 9$, $7 \cdot 2,6 \cdot 6,6,5,4 \cdot 4$. The values of the differences of $\log y$ at the extremities of the stretches of $x 80-160,120-200,180$ 260 , and $220-300$, were respectively proportional to 23,18 , 16 , and 13. There is no approach to constancy, although the average decrease of the range per oscillation during the last 40 oscillations was only one three-hundredth part of the average range during these oscillations.

The point will be considered further subsequently.

\section{Approximate Isochronism of the Oscillations.}

An experiment was made, immediately after the performance of experiment $M$, to determine the period of oscillation over different ranges. The numbers in the first column below, when multiplied by 5 , give the initial scalereadings : in the second column are given the corresponding times occupied by the next ten oscillations, the unit being the second. In addition the zero was occasionally read, the values being given in the third column.

\begin{tabular}{|c|c|c|c|c|c|}
\hline $\begin{array}{r}5 \cdot 48 \\
5.84 \\
5 \cdot 30 \\
7 \cdot 15 \\
6.05 \\
5.45 \\
14.90 \\
6.60 \\
11.80 \\
7 \cdot 60 \\
13.20 \\
7.65 \\
6.08\end{array}$ & $\begin{array}{l}79 \cdot 0 \\
78 \cdot 8 \\
78 \cdot 5 \\
79 \cdot 0 \\
78 \cdot 5 \\
78 \cdot 5 \\
79 \cdot 7 \\
78 \cdot 5 \\
795 \\
79 \cdot 1 \\
80 \cdot 0 \\
80 \cdot 0 \\
79 \cdot 8\end{array}$ & $\begin{array}{l}3.36 \\
3.36\end{array}$ & $\begin{array}{r}5 \cdot 50 \\
5 \cdot 12 \\
4 \cdot 95 \\
13.90 \\
8 \cdot 00 \\
6 \cdot 15 \\
4 \cdot 80 \\
4 \cdot 50 \\
16.50 \\
7 \cdot 80 \\
4 \cdot 07 \\
17.00 \\
7 \cdot 50 \\
4.28\end{array}$ & $\begin{array}{l}78 \cdot 4 \\
79 \cdot 0 \\
78 \cdot 8 \\
79 \cdot 8 \\
79 \cdot 4 \\
79 \cdot 0 \\
78 \cdot 5 \\
78 \cdot 5 \\
80 \cdot 2 \\
79 \cdot 3 \\
79 \cdot 2 \\
80 \cdot 4 \\
79 \cdot 5 \\
78 \cdot 8\end{array}$ & $\begin{array}{l}3.49 \\
3.49\end{array}$ \\
\hline
\end{tabular}

Despite the irregularities inseparable from the conditions of the experiment, it is evident that the period, while nearly constant, increases slightly as the range increases.

\section{Damping due to the Viscosity of Air.}

In one experiment the surface of the oscillating part of the apparatus was more than doubled by placing light sheets of rongh paper on the top of the lead ring. It was found that this did not produce the slightest difference in the observed results. Thus the decay of the oscillations is not appreciably affected by the viscosity of the air. 


\section{Kelvin's and Wiedemann's Theories.}

Lord Kelvin said that the decay of the oscillations might be due to viscous resistance, i.e. resistance to change of shape depending on the rate of change, in which case the elasticity is perfect within the limits of the experiment. On the other hand he remarked that, if it were due to dependence of the elastic resilient force on previous conditions of strain, the phenomenon would be continuous with imperfectness of elasticity, and this was indicated to some extent by the phenomenon of "fatigue."

His final experiments were made with small distortions, within the palpable limits of elasticity, to test the former thieory. The loss of energy per vibration was greater than could be accounted for by dissipation ensuing on change of shape of an elastic solid, and it was also immensely greater than that due to resistance of the air. He considered also that the results showed a loss of energy much greater than any that could be accounted for by imperfection of elasticity.

Though the compound-interest law was precisely that which would arise from viscous resistance, yet, in that case, the logarithmic decrement should vary inversely as the period; but this was far from being so. Tomlinson finds that the decrement is nearly independent of the period in the more "non-viscous" metals, and that it increases markedly with the period in other metals. Kelvin remarked that the result was such as might be due to "after-action" or imperfection of elasticity.

Wiedemann's statical observations showed that a wire which had once been twisted behaved in quite different ways according as it was twisted to one side or the other of its existing position of equilibrium; whence he concluded that "the hypothesis according to which the decay of torsional oscillations is due to internal friction depending on the velocity alone, can no longer be maintained." Nor could the elastic after-action alone account for it. He then stated his own theory as follows:-Let the wire be "accommodated" by frequent rotations to and fro (see the account of his experimental results on p. 37), and therein at last be temporarily twisted in the positive direction, describing an angle $+a$, while the molecules may be rotated so that the lower ends of their axes, looked at from the axis of the wire, describe an angle $+\alpha$, say to the left. The direction of this rotation follows from my magnetic experiments. If the wire be slowly brought back into the permanent torsion-position $+b$, the axes of the molecules will retain a portion $+\beta$ of their rota- 
tion to the left. If the wire now receives an impulse in the positive direction, which again elongates it up to $+a$, according to the laws of perfect elasticity it will swing back again to the position $+b$. If it now swings beyond this position farther to the right, and if the molecules in their rotation had no friction on one another at all to overcome, it would arrive at the elongation $-a$, since the same force that twists it from $+b$ to $+a$ twists it in the contrary direction from $+b$ to $-a$, while the axes of the molecules would be rotated just as far $(-\alpha)$ to the right as previously to the left. Again, with perfect elasticity the wire would go back to the position $-b$, in which the molecules would be rotated $-\beta$, and so forth. The to-and-fro motions of the wire, between $\pm a$ and $\pm b$, are perfectly elastic; therefore the performances of work in the swingings outwards and the swingings back again must, within these limits, completely compensate one another. In fact, however, there results a diminution of the amplitudes of oscillation; hence the loss of vis viva therein can only correspond to the work which is expended for the alteration of the positions of equilibrinm, or the rotation of the molecules from $+\beta$ to $-\beta$, which determine it.

\section{Theory of the Present Results.}

The kinetic theory of the viscosity of gases, as developed by Maxwell, asserts that viscosity is due to interchange of momentum between relatively-moving portions of the substances-this interchange being effeeted by the passage of molecules from one portion to the other. In the same way the viscosity of liquids is explained. There is essentially a passage of molecules from one group to another. Such passage does not take place in a perfectly elastic solid; but there may still be interchange of momentum in the relative motion of the constituents of a group, and therefore true viscosity in a solid. Yet, if the potential energy of deformation of a group is large in comparison with the kinetic energy of average relative motion of the constituent molecules of the group-a condition which holds in the case of the torsional vibrations of a fine metallic wire to the free end of which is attached a mass of great moment of inertia-it seems certain that the energy dissipated by true viscosity will be small in comparison with the energy dissipated in the breaking down of molecular groups (as in 'Maxwell's theory of a molecularly constituted solid), should such rupture take place to, possibly, a swall extent only.

Wiedemann seems to regard the loss of energy as due to the work done in rotating the molecules from one position of 
stable equilibrium to another, that rotation causing, in its turn, the permanent relative displacement of the molecules which occurs in the "set :" and this, of course, is quite a plausible hypothesis. In the homogeneous permanent torsional strain the final configuration of a given group of molecules is essentially similar to the initial configuration; yet we may, since a position of unstable equilibrium has been passed, say that the old configuration has broken down and that a new one has been formed; so that the loss of energy may be ascribed to the work done in breaking down molecular configurations.

There seems to me to be no need to ascribe such a special type to the rupture of the configurations. On the contrary, I think it more likely that the permanent molecular rotation is a consequence of the permanent set which ensues on complete rupture of configurations-i.e. rupture in which molecules in given configurations part company, new groups being formed. It appears to be most probable that the loss of energy in this total rupture greatly exceeds, group by group, the loss due to the cause which Wiedemann contemplates.

Such total rupture takes place in all liquids and in plastic solids; and, by analogy, we conclude that it takes place in all solids to a greater or less extent. Indeed we do not need to rest upon analogy. The evaporation of ice below the freezing-point is due to such rupture ; and so, probably, as has been remarked, is the characteristic smell of metals, such as freshly cut copper or iron. At a given temperature, the molecules in a given configuration are in rapid motion with a certain average speed, the actual speed of a molecule being sometimes greatly in defect, sometimes greatly in excess, of this average. When the excess is sufficiently great, the molecule will pass beyond the range of the molecular action of the remaining constituents of the group, rupture of the configuration will occur, and the potential energy of deformation will be transformed into heat energy. In a sufficiently long interval of time such rupture must occur, according to the kinetic theory. In a given state of strain it occurs more readily the higher the temperature; at a given temperature it occurs more readily the greater the strain.

It is easy to see that, if a molecule is under directional control by molecular forces, rotation will in general ensue on rupture of a given group: A dynamical illustration will make this plain.

Let $P Q$ represent a pendulum, with a massive bob $Q$, supported at the point $P$. Let the pendulum be situated 
symmetrically between the rigid fixed blocks $A, A^{\prime}$, to which it is attached by equal and similar elastic cords $l, l^{\prime}$; and let the pendulum be attached also by equal and similar elastic cords $\lambda, \lambda^{\prime}$, to the rough movable blocks $B, B^{\prime}$, which rest, symmetrically with regard to $\mathrm{PQ}$, upon $\mathrm{A}, \mathrm{A}^{\prime}$.' So long as $\mathrm{B}, \mathrm{B}^{\prime}$ are not displaced the equilibrium position of $\mathrm{PQ}$ will not be altered; but if the bob be moved to the right to such an extent as to move the block $B$ into the position indicated by the dotted lines, so that the cord $\lambda$ becomes slacker, the

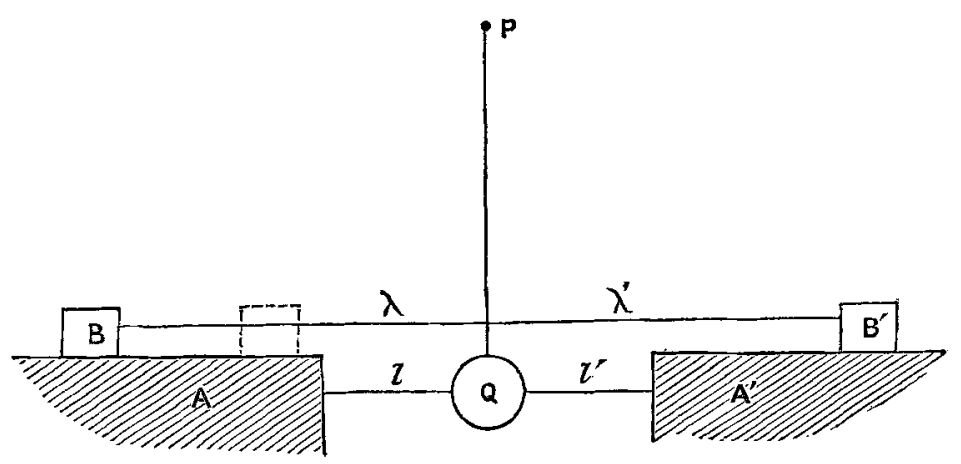

equilibrium position will change. The slackening of the cord $\lambda$ corresponds to the removal of a molecule from a given configuration. The displacement of the equilibrium position corresponds to the set of the molecule $\mathrm{PQ}$. There is change of relative position of $\mathrm{Q}, \mathrm{A}, \mathrm{A}^{\prime}$, and $\mathrm{B}^{\prime}$, and there is also rotation of $\mathrm{PQ}$ from its old direction. The change which takes place in one group is shared by all other groups which are in direct or indirect connexion with that group. This could be shown in the model by attaching $P Q$ by elastic cords to other pendulums. In this way the whole system takes a set.

Displacement of $Q$ from the new position of equilibrium towards the right will take place strictly in accordance with Hooke's Law so long as the former maximum displacement is not exceeded. The old maximum force will be needed to cause the original maximum displacement to the right, but the actual displacement which it causes is now measured from the new equilibrium position. An equal force applied in the opposite direction shifts the equilibrium point back to its old situation, and gives a maximum displacement to the left, from this point, equal to the former maximum to the right. As regarded from the second equilibrium position to the right, the system resists more strongly displacements farther to the

Phil. Mag. S. 5. Vol. 38. No. 230. July 1894. E 
right than it resists equal displacements to the left. These facts illustrate Wiedemann's experimental results with regard to the torsion of wires.

Consider the wire in its initial undisturbed condition, or in any condition of equilibrium with set provided that the next torsion is to be oppositely directed to that which was last performed and which produced the set. If the wire be now twisted through an angle $\theta$, and if, during that twist, no configurations break down, the potential energy will be represented, in accordance with Hooke's Law, by the expression

$$
\mathrm{V}=\frac{1}{2} k \theta^{2}
$$

where $k$ is a constant. If there is rupture of molecular groups, the potential energy will fall short of the above amount by a quantity which we shall assume to be proportional to a power of the angle. Thus we get

$$
\mathrm{V}=\frac{1}{2} k \theta^{2}-p \theta^{m}
$$

In those cases in which the loss of energy, per outward swing, is small in comparison with the total energy, so that the set is negligible in comparison with the total range, the loss is practically equal to $k \theta d \theta$. Hence (3) takes the form

$$
-k \theta d \theta=p \theta^{m} d t
$$

where $d t$ is the time of an outward swing. The integral is

$$
\theta^{n}\left(t+t_{0}\right)=b, \text {. . . . . . }
$$

where $b$ and $t_{0}$ are constants, and $n=m+2$.

This is exactly our empirical equation (1); which we thus derive as an approximate consequence of the theory that the loss of energy is due to the rupture of molecular configurations, and is proportional to a power of the angle of torsion.

A glance at the curves in fig. 1 will show that, in the steeper portions, the drop of angle per single swing is not really negligible in comparison with the range, though, even in the steepest portions, it does not exceed one tenth part of the range and rapidly diminishes as the range decreases. Hence we cannot expect the values of $n$ which hold throughout those portions of the curves which are dealt with in Table II. to hold at still smaller angles of distortion. The value of $n$ which holds approximately over a considerable stretch of a curve where the angle is large will, quite apart from after-action, be greater than the value which holds over a considerable stretch where the angle is small.

\section{Deduction of the Compound-Interest Law.}

In experiment $C$ the value of $n$ decreased, as time went on, from a value greater than unity to a value less than unity. 
If, in equation (4), we put $m=2$, equation (5) takes the form

$$
\theta=\theta_{0} \epsilon^{-a t} \text {, }
$$

which expresses the above law. This law therefore follows from our theory if we suppose that $n$ decreases in value to the limit zero; and the theory shows that the decrement of energy per swing then follows the compound-interest lawjust as the decrement of angle does.

\section{Explanation of After-action.}

When a wire is held in a state of torsion under a constant couple, some of the less stable molecular groups will in time break down, and so the strain slowly increases. If it be held in a given state of strain, this gradual rupture of groups necessitates a slow diminution of the couple. On the removal of the couple, the wire remains in a state of internal stress because of the set. Consequently the gradual rupture of groups produces a slow diminution of set; for the strongest groups remain unbroken in the original deformation, and, in any ordinary experiment, the groups which break form a small fraction of the whole. This is Maxwell's explanation.

The after-action takes place with comparative rapidity at first: afterwards it goes on more slowly. It takes place more and more completely the longer the strain is continued, and requires proportionally longer maintenance of an equal reverse strain to undo it. Hence, if a wire be twisted first to the right through a given angle for a long time, then to the left through an equal angle for a short time, and be then gradually put into the position of set, we should expect that the set would change (as it does) first in the sense of recovery from the second strain, and finally in the sense of recovery from the first strain.

\section{Conditions of Maximum and of Zero Resilience.}

From equation. (3) we obtain

$$
\begin{array}{rlrl} 
& -\frac{d \mathrm{~V}}{d \theta} & =-k \theta+m p \theta^{m-1}, \\
& =-k \theta+k^{\prime} \theta^{n+1}, \\
\text { and } & & -\frac{d^{2} \mathrm{~V}}{d \theta^{2}} & =-k+k^{\prime}(n+1) \theta^{n} .
\end{array}
$$

Hence we see that there is angle of maximum resilience given by

$$
\bar{\theta}^{n}=\frac{k}{k^{\prime}} \frac{1}{(n+1)}, \cdots \cdot \ldots
$$


and a condition of zero resilience indicated by

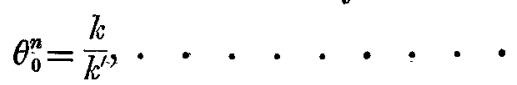

the relation between $\bar{\theta}$ and $\theta_{0}$ being

$$
\theta_{0}=\bar{\theta}(n+1)^{\frac{1}{n}} \text {. }
$$

As the torsion of a wire is increased, the set and the difference between the angle of torsion and the angle of set increase. This goes on until the angle $\bar{\theta}$ is reached. As the twisting couple is further increased, the set increases at a greater rate than the torsion. The stronger configurations now break down, and the removal of the twisting couple is followed by small recoil. If the twisting couple be maintained in excess of the value required to overcome the maximum resilient couple, work is done constantly in breaking up molecular groups, and the material of the wire flows steadily, the angle of torsion and the set increasing at constant equal rates. Under that constant couple there is also constant resilience. The condition corresponding to the theoretical angle $\theta_{0}$ is attainable under a finite couple of moment $k^{\prime} \theta_{0}^{n+1}=k \theta_{0}$. Thus the theory indicates that the melting-point is conditioned by shearing-stress.

The flow will of course commence at the surface of the wire. The angle $\bar{\theta}$ might also be called the Angle of Plasticity, and the couple $k \theta_{0}$ might be termed the Couple of Fluidity,

\section{Relation between Torsion and Set.}

If we assume that the torsional rigidity is not sensibly altered by set, the quantity $k$ is constant, and we may write (3) in the form

$$
\frac{1}{2} k(\theta-\alpha)^{2}=\frac{1}{2} k \theta^{2}-p \theta^{m},
$$

where $\alpha$ is the angle of set. This gives

$$
\alpha=\theta\left(1-\sqrt{\left.1-2 \frac{k^{\prime}}{k} \frac{\theta^{n}}{n+2}\right)}\right. \text {. }
$$

To test this expression I have used Wiedemann's statical observations given in Table I. p. 4, Phil. Mag. 1880, vol. ix. I find that the equation

$$
\alpha=\theta\left(1-\sqrt{1-896(10)^{-16} \theta^{3 \cdot 548}}\right)
$$

corresponds to a remarkable extent with his observations. The curve in fig. 2 represents this equation with values of $\theta$ as ordinates and values of $\alpha$ as abscissæ; and the points on or near it represent Wiedemann's results. No stronger confirmation of the theory need be desired. 


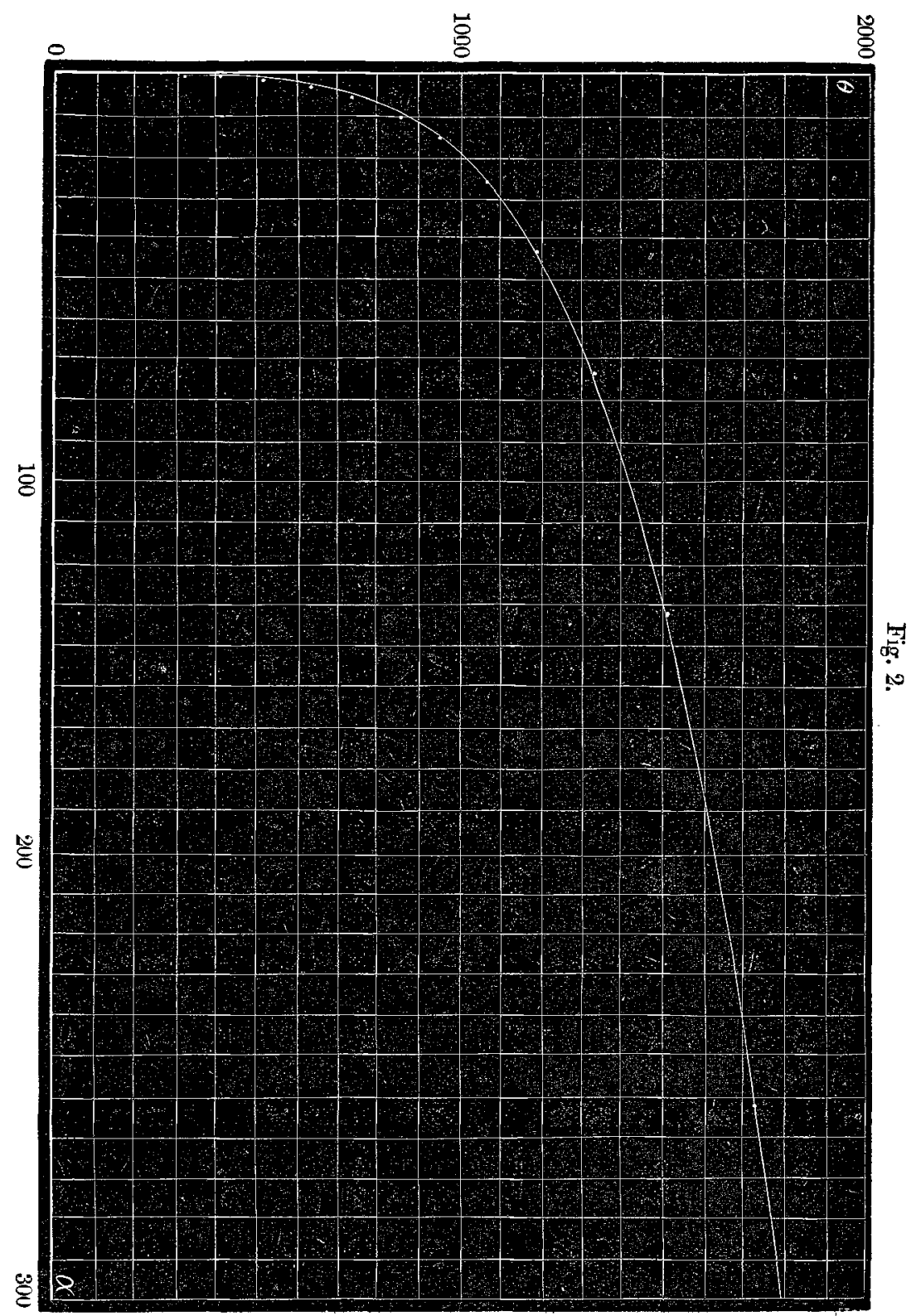




$$
\text { Period of Oscillation. }
$$

The potential energy of the system is

$$
\mathrm{V}=\frac{1}{2} k \theta^{2}-p \theta^{m} \text {. }
$$

The kinetic energy of the system oscillating as a whole is

$$
\frac{1}{2} \mathrm{I} \dot{\theta}^{2}
$$

where $I$ is the moment of inertia; and the second term in the expression for $V$ represents kinetic energy of molecular motion. So the total kinetic energy at the angle $\theta$ is

Hence we have

$$
\mathrm{T}=\frac{1}{2} \mathrm{I} \dot{\theta^{2}}+p \theta^{m} \text {. }
$$

$$
\mathrm{I} \dot{\theta}^{2}+k \theta^{2}=\text { constant, }
$$

which shows that the motion outwards is simple harmonic motion as reckoned from the origin; but it is only so in virtue of the condition that the defect of the potential energy from the value that it would have in accordance with Hooke's Law is due to its transformation into a kinetic form. The periods of the outward swing from zero and of the inward swing to the position of set, on the assumption that $k$ does not change, are each equal to

$$
\frac{\pi}{2} \sqrt{\frac{I}{k}}
$$

Wiedemann's statical experiments show that after the few preliminary applications of the maximum twisting couple necessary to fix the set, $\theta-\alpha$ varies almost in accordance with Hooke's Law, and that the slight difference is in the direction of too great magnitude as the torsion increases: and Tomlinson has shown that great permanent torsion decreases the torsional elasticity. These facts may indicate that $k$ is slightly decreased at the greater torsions, in which case the period of oscillation will slightly increase as the range is increased.

\section{Concluding Remarks.}

The experiment A was not the first made with the given wire, though it was the first made with it under the stated conditions of length \&c. Thus, in A the wire was in a fatigued condition relatively to its condition in the experiments $R$ and $\mathrm{S}$.

It has been found by Kelvin and Tomlinson that, in the case of small ranges, the rate of decrease of range per oscillation is practically constant for all periods of oscillation in 
the less viscous metals and increases with the period in other metals. According to the above theory this is due to the fact that a given state of stress is continued longer, so that the molecular configurations have more opportunity to break down.

If the theory were pushed to the extreme in its application to Wiedemann's results on torsion and set above quoted, we. should find that $\bar{\theta}=2400$, and that the couple necessary for zero resilience was fully double the maximum couple employed by Wiedemann (that corresponding to $\theta=1725$ ).

Various deductions might be drawn from the theory in connexion with the observed values of the constants in the empirical equations. I do not think that such deductions would be of any value except in connexion with a much wider experimental basis than that furnished above. I hope soon to be able to communicate the results of further observations.

IV. On the Mechanism of Electrical Conduction.-Part I. Conduction in Metals. By Charles V. Burton, D.Sc.*

1. $T$ THE view of electrical conduction which it is here my object to explain receives general support from more than one consideration; for it leads to the conclusion that deviations from Ohm's Law must be quite inappreciable in the case of metallic conductors, and it goes far to explain, I think, why metals are so much less opaque than thei ${ }_{t}$ ordinary conductivities would lead us to infer. But it is no alone on such considerations that wo have to rely, for, as $i^{t}$ seems to me, the main conclusions are capable of exact demonstration ; and accordingly it would appear most convenient to commence with a few simple theorems, seeking afterwards to account for known phenomena by means of our definite results.

\section{TheOREM I.}

In a region containing matter, there may be (and probably always are) some parts which are perfect insulators and some parts which are perfect conductors; but there can be no parts whose conductivity is finite-unless every finitely conductive portion is enclosed by a perfectly conductive envelope.

Before proceeding to the proof of this theorem, it may be remarked that the presence of the last clause in no way modifies any application of our result, since the space within a perfectly conductive envelope is completely shielded from

* Communicated by the Physical Society : read April 13, 1894. 